\title{
Sliding mode control for active vibration isolation of a long range scanning tunneling microscope
}

\author{
Kuo-Jung Lan and Jia-Yush Yen \\ Department of Mechanical Engineering, National Taiwan University, Taipei, Taiwan 10617 \\ John A. Kramar \\ National Institute of Standards and Technology, Gaithersburg, Maryland 20899
}

(Received 5 March 2004; accepted 26 July 2004; published 29 October 2004)

\begin{abstract}
An active vibration isolation (AVI) system has been designed and implemented for the Molecular Measuring Machine $\left(\mathrm{M}^{3}\right)$ at the National Institute of Standards and Technology (NIST). NIST is investigating active vibration isolation as an approach to improving the $\mathrm{M}^{3}$ image resolution and measurement speed. This article presents the full dynamic model of the AVI system with the Mallock suspension used for the $\mathrm{M}^{3}$ system suspension. A decoupling process is employed to decompose the complicate dynamics into separate axis. This article then applied a sliding mode controller (SMC) to overcome the system nonlinearities. Experimental results show that the controller is effective, achieving a vibration attenuation of $10 \mathrm{~dB}$ at some frequencies, depending on the axis. () 2004 American Institute of Physics. [DOI: 10.1063/1.1807005]
\end{abstract}

\section{INTRODUCTION}

The Molecular Measuring Machine $\left(\mathrm{M}^{3}\right)$ at the National Institute of Standards and Technology (NIST) is an instrument for measuring feature coordinate in two dimensions, designed to achieve subnanometer resolution over a $50 \mathrm{~mm}$ $\times 50 \mathrm{~mm}$ area. Three are many challenging engineering consideration for such an instrument, including those dealing with isolation from environmental noise sources. To limit vibration noise from external sources, the $\mathrm{M}^{3}$ uses a twostage, passive vibration isolation support system. ${ }^{1,2}$

Now to improve the system performance, NIST has installed six degree-of-freedom active vibration isolation (AVI) system. ${ }^{3}$ The AVI system in $\mathrm{M}^{3}$ uses five piezoelectric actuators (PZT) as the lateral-constraint links in a Mallock suspension system, and a sixth piezo actuator to control the vertical movement. Although there are many articles regarding vibration isolation in the literature, ${ }^{4-6}$ the NIST system requires extremely high performance. The level of disturbance transmitted from ground to the measuring system must be attenuated below a nanometer. Also, the highly nonlinear Mallock suspension system identification and the subsequent controller design very challenging.

This article develops and describes the implementation of a controller for the AVI system. First, a mathematical model is derived by using a spring-damper model. Then, a decoupling procedure is used to reduce the coupling terms in the transfer function matrix. The system decoupling is very helpful in reducing the system complexity so that real-time control is realizable. A six-by-six Multiple-Input-MultipleOutput (MIMO) system can be reduced to six Single-InputSingle-Output (SISO) systems. Next, a system identification of the Mallock suspension is developed, using curve fitting on the diagonalized system. The inner Mallock shell itself is treated as a rigid body. The model serves as the basis for the design of a robust Sliding Mode Controller (SMC). This ar- ticle presents the standard (SMC) control, considering the system parameter uncertainties and exogenous disturbances. We also use the Lyapunov function to verify the stability of the controller. SMC offers a high degree of robustness against model uncertainty; therefore it is the most suitable for dealing with the nonlinearities and the unmodeled dynamics in the system. Furthermore, the simulation shows that the SMC provides good performance near $100 \mathrm{~Hz}$. The experimental results show that the controller achieves a $5 \mathrm{~dB}$ to $10 \mathrm{~dB}$ vibration attenuation in the desired bandwidth.

This article is divided into seven major sections. Section II describes mechanical arrangement of the Mallock suspension system in $\mathrm{M}^{3}$. Section III presents the system identification process. Section IV describes the controller design and the stability analysis. Section V presents the simulation results. Section VI presents the experimental implementation.

\section{THE $M^{3}$ ACTIVE VIBRATION ISOLATION SYSTEM}

The Molecular Measuring Machine uses a Mallock suspension system for vibration isolation. The Mallock system contains an inner and an outer supporting shell linked by six rods and one spring as shown in Fig. 1. ${ }^{7}$ Figure 2 shows a photograph of the inner and outer shells.

The first, second, and third rod are oriented in the $x$ direction and are parallel to each other (see Fig. 3). The fourth and fifth rod are oriented in the $y$ direction, and the sixth rod and the suspension spring are parallel to each other and oriented in the $z$ (vertical) direction. Therefore the first, second, and third rod control the movement in the $x$ direction; the fourth and fifth rod control the $y$ direction movement; and the $z$ direction position is determined by the sixth rod. The suspension spring is connected to a point which is horizontally offset from the center-of-mass axis; the resulting torque moment preloads the suspension rods in slight com- 


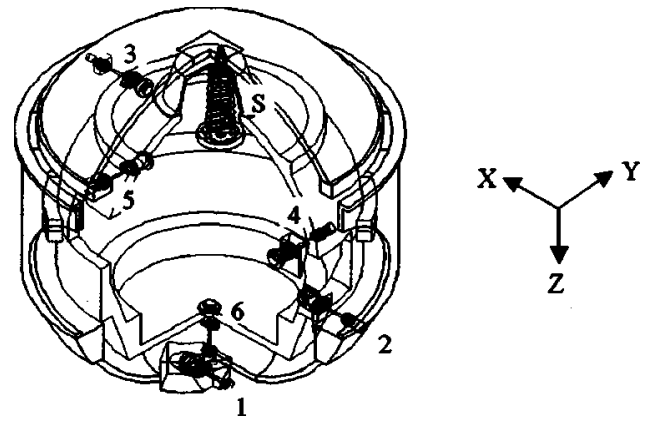

FIG. 1. A diagram for the Mallock suspensin system of $\mathrm{M}^{3}$. Actuators are labeled by number and interconnect the Mallock inner and outer shell. Suspension spring in $\mathrm{S}$ and supports the Mallock inner shell. The $\mathrm{M}^{3}$ measurements system is placed inside the inner shell, which is not shown in the figure.

pression. Additional spring preloads are used in parallel with each actuator to increase the coupling stiffness and the robustness of the suspension. ${ }^{8}$ The first, second, and third rods mainly determine the yaw and pitch orientation; the fourth and fifth rods determine the roll orientation (about $x$ ). Because the rods are not orthogonal to each other, the Mallock suspension system has serious cross-axis coupling.

Cross coupling is unavoidable in any parallel-linkage suspension system, including the more familiar Stewart platform. ${ }^{5}$ Still, these systems are widely used because they are in principle stiffer than sequentially stacked systems with comparable degrees of freedom.

\section{SYSTEM MODELING AND IDENTIFICATION}

For system modeling, we use the nomenclature and coordinate system defined in Fig. 3. Applying the NewtonEuler equation to the Mallock inner shell, we have

$$
\begin{aligned}
& \sum \mathbf{F}=m \dot{\mathbf{v}}_{b}+\mathbf{w}_{b} \times m \mathbf{v}_{b}, \\
& \sum \mathbf{M}=\mathbf{I} \dot{\mathbf{w}}_{b}+\mathbf{w}_{b} \times \mathbf{I} \mathbf{w}_{b},
\end{aligned}
$$

where $\mathbf{v}_{b}$ is the velocity vector and $\mathbf{w}_{b}$ is the body angular rate. The mass of Mallock inner shell is $m$, and $\mathbf{I}$ is the moment of inertia matrix. $\Sigma \mathbf{F}$ is the net force vector on inner shell and $\Sigma \mathbf{M}$ represents the net moment vector shown in Eq. (3),

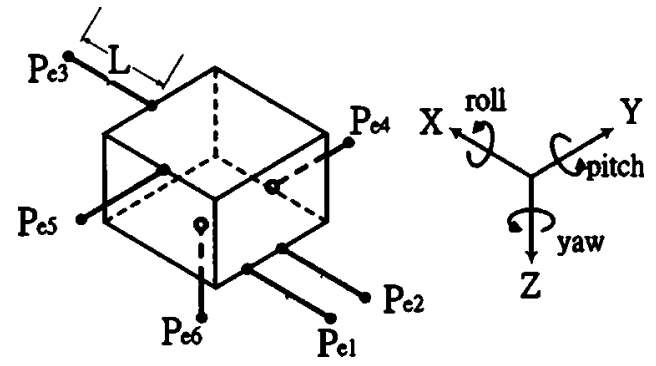

FIG. 3. Mallock suspension system. Pei represents the $i$ th actuator and L is the actuator length. The cube represents the Mallock inner shell.

$$
\begin{aligned}
& \sum \mathbf{F}=\sum_{i=1}^{6} \mathbf{R}^{T} \mathbf{F}_{i}+\mathbf{R}^{T} \mathbf{F}_{s}+\mathbf{R}^{T} m \mathbf{g}, \\
& \sum \mathbf{M}=\sum_{i=1}^{6} \mathbf{D}_{i} \times \mathbf{R}^{T} \mathbf{F}_{i}+\mathbf{D}_{s} \times \mathbf{R}^{T} \mathbf{F}_{s},
\end{aligned}
$$

where $\mathbf{D}_{i}$ and $\mathbf{D}_{s}$ are the displacement vector from the center of gravity $(\mathrm{cg})$ of the body to the $i$ th piezoelectric linkage and the sping and point, respectively. $\mathbf{F}_{i}$ is the force vector generated by each rod, $\mathbf{F}_{s}$ is the force vector generated by the suspension spring, $m \mathbf{g}$ is the gravitational force, and $\mathbf{R}$ is the rotation matrix. The superscript $T$ means the matrix transformation. We model $\mathbf{F}_{i}$ as a spring and a damper, as shown in Eq. (4),

$$
\mathbf{F}_{i}=-\mathbf{K}_{r i} \Delta \dot{\mathbf{x}}_{e i}-\mathbf{C}_{r i} \Delta \dot{\mathbf{x}}_{e i} \quad(i=1, \ldots, 6),
$$

where the vector $\mathbf{K}_{r i}$ is the spring constant of the Viton rubber pads in earth coordinates for channel $i$, the $\mathbf{C}_{r i}$ is the damping constant of the Viton rubber pads for channel $i, \Delta \mathbf{x}_{e i}$ is the extension length of spring $i$, and $\Delta \dot{\mathbf{x}}_{e i}$ is the extension rate of spring $i$. The value of the vector $\mathbf{K}_{r i}$ was described in above section. The $\Delta \mathbf{x}_{e i}$ means the displacement from the end of the PZT to the jointed point on the Mallock inner shell, which can be expressed as

$$
\Delta \mathbf{x}_{e i}=\mathbf{p}_{i}-\mathbf{p}_{s i} \quad(i=1, \ldots, 6),
$$

where $\mathbf{p}_{s i}$ is the location at one end of the PZT for channel $i$, $\mathbf{p}_{c}$ is the mass of the Mallock inner shell, and $\mathbf{p}_{i}$ is the jointed point on the Mallock inner shell, shown in Fig. 4,

$$
\mathbf{p}_{s i}=\mathbf{p}_{e i}+\mathbf{R}_{p i} \overrightarrow{\mathbf{U}}_{i} \cdot\left(L+u_{i}\right),
$$

where $\overrightarrow{\mathbf{U}}_{i}$ is initial unit vector pointing from $\mathbf{p}_{e i}$ to $\mathbf{p}_{s i}$ in body coordinates, $\underline{L}$ is the original length of the PZT, and $u_{i}$ is the deflection of PZT $i$ due to the applied voltage. If the initial

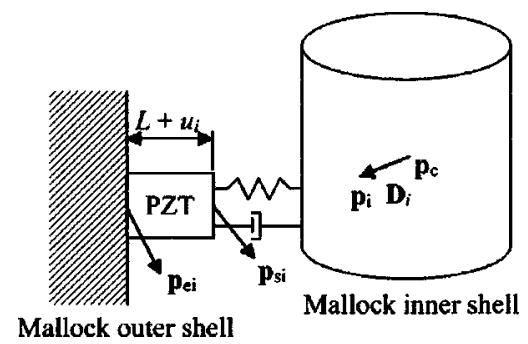

FIG. 4. The schematic diagram of the $i$ th PZT and Viton rubber system. The Viton rubber is simulated as a spring and a damper. 
status for each rod is horizontal for channels 1-5 and vertical for channel 6 , the $\overrightarrow{\mathbf{U}}_{i}$ can be described as follows:

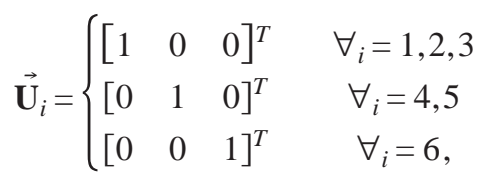

where the superscript $\mathrm{T}$ means the matrix transformation. A linearized system is used in the controller design. We define the system states as

$$
\left[\begin{array}{llll}
\mathbf{x}_{e}^{T} & \theta_{e}^{T} & \mathbf{v}_{b}^{T} & \mathbf{w}_{b}^{T}
\end{array}\right]^{T},
$$

where $\mathbf{x}_{e}$ is the displacement of inner shell in the earth coordinate frame. The state space form of the system becomes

$$
\begin{aligned}
& \dot{\mathbf{x}}_{e}=\widetilde{\mathbf{R}} \mathbf{v}_{b}, \\
& \dot{\theta}_{e}=\widetilde{\mathbf{T}} \dot{\mathbf{w}}_{b}, \\
& \dot{\mathbf{v}}_{b}=\frac{1}{m} \sum \mathbf{F}, \\
& \dot{\mathbf{w}}_{b}=\mathbf{I}^{-1} \sum \mathbf{M},
\end{aligned}
$$

where $\tilde{\mathbf{R}}$ is linearized rotation matrix and $\widetilde{\mathbf{T}}$ is linearized rotation-rate matrix $\theta_{e}$ is the Euler angle.

System diagonalization: To simplify the implementation of the controller, we diagonalize the system. As a heuristic example, consider a two-input-two-output system. Given a transfer function, $G_{i j}(s)$, and the Laplace transform of the inputs, $U_{i}(s)$, the outputs are the product,

$$
\left[\begin{array}{l}
Y_{1}(s) \\
Y_{2}(s)
\end{array}\right]=\left[\begin{array}{ll}
G_{11}(s) & G_{12}(s) \\
G_{21}(s) & G_{22}(s)
\end{array}\right]\left[\begin{array}{c}
U_{1}(s) \\
U_{2}(s)
\end{array}\right] .
$$

If one transforms the system by pre and postmultiplying by a diagonalization matrix, the system becomes

$$
\begin{aligned}
{\left[\begin{array}{c}
\bar{Y}_{1}(s) \\
\bar{Y}_{2}(s)
\end{array}\right]=} & {\left[\begin{array}{cc}
a & b \\
c & -d
\end{array}\right]\left[\begin{array}{ll}
G_{11}(s) & G_{12}(s) \\
G_{21}(s) & G_{22}(s)
\end{array}\right]\left[\begin{array}{cc}
e & f \\
g & -h
\end{array}\right] } \\
& \times\left[\begin{array}{c}
\bar{U}_{1}(s) \\
\bar{U}_{2}(s)
\end{array}\right],
\end{aligned}
$$

$$
\left[\begin{array}{l}
\bar{Y}_{1}(s) \\
\bar{Y}_{2}(s)
\end{array}\right]=\left[\begin{array}{ll}
\bar{G}_{11}(s) & \bar{G}_{12}(s) \\
\bar{G}_{21}(s) & \bar{G}_{22}(s)
\end{array}\right]\left[\begin{array}{c}
\bar{U}_{1}(s) \\
\bar{U}_{2}(s)
\end{array}\right],
$$

where $a, b, c, d, e, f, g$ and $h$ are positive constants to be determined such that the determinant of diagonalized matrix is not zero. We determine these constants by maximizing the diagonal terms $\left(\bar{G}_{11}, \bar{G}_{22}\right)$ and minimizing the off diagonal terms $\left(\bar{G}_{12}, \bar{G}_{21}\right)$. Because $G i j(j w)$ is a transfer function, which means it is a complex value and frequency-dependent, it is impossible to find a constant diagonalized matrix to reduce the coupling effect for all of the frequency range. Therefore, the experiment only minimizes the coupling effect near the operating frequency, $w_{0}$. We chose a frequency $w_{0}$ $(100-200 \mathrm{~Hz})$ to minimize the off-diagonal terms. The solution is shown in Eq. (13),

$$
\begin{aligned}
& a=c=1 /(1+q), \quad b=d=1-a, \\
& e=f=1(1+p), \quad g=h=1-e,
\end{aligned}
$$

We chose the frequency $w_{0}$ at the peak value of the offdiagonal terms $\left(G_{i j}, G_{j i}\right)$. After diagonalization, the magnitude of the off-diagonal terms $\left(\bar{G}_{i j}, \bar{G}_{j i}\right)$ near $w_{0}$ will be reduced. We diagonalize channels one, two, and three as channels $X, \theta$, and $\psi$; and we convert channels four and five to channels $Y$ and $\phi$. Channel 6 remains the same, but its notation is changed to channel $Z$. This process is performed on the actual system to obtain a diagonal system transfer function. it is important to note that the solution only minimizes the off-diagonal terms near $w_{0}$.

Figure 5 is the Bode diagram or frequency response of the original system as measured by a frequency analyzer. Each piezo actuator is driven in turn, and for each, the re- sponse is measured at all six accelerometer sensors. In contrast, Fig. 6 shows the Bode diagram of the diagonalized system. One easily observes from Fig. 5 that there are serious coupling effects, especially between the $(1,2)$ and the $(2,1)$ entries, and between the $(4,5)$ and $(5,4)$ entries. The nondiagonal entries in Fig. 6, on the other hand, are up to $20 \mathrm{~dB}$ smaller than the diagonal entries. The curve-fit models we generated for the diagonal terms in the resulting system are shown in Fig. 7 as the solid lines. The dashed lines are the frequency response of the real system as taken by a frequency analyzer. Because the signal to noise ratio of subsystems $X$ and $Y$ at low frequency range is small, the data at that region cannot be trusted. However the input signal represents a position actuator and the output signal is the accel- 


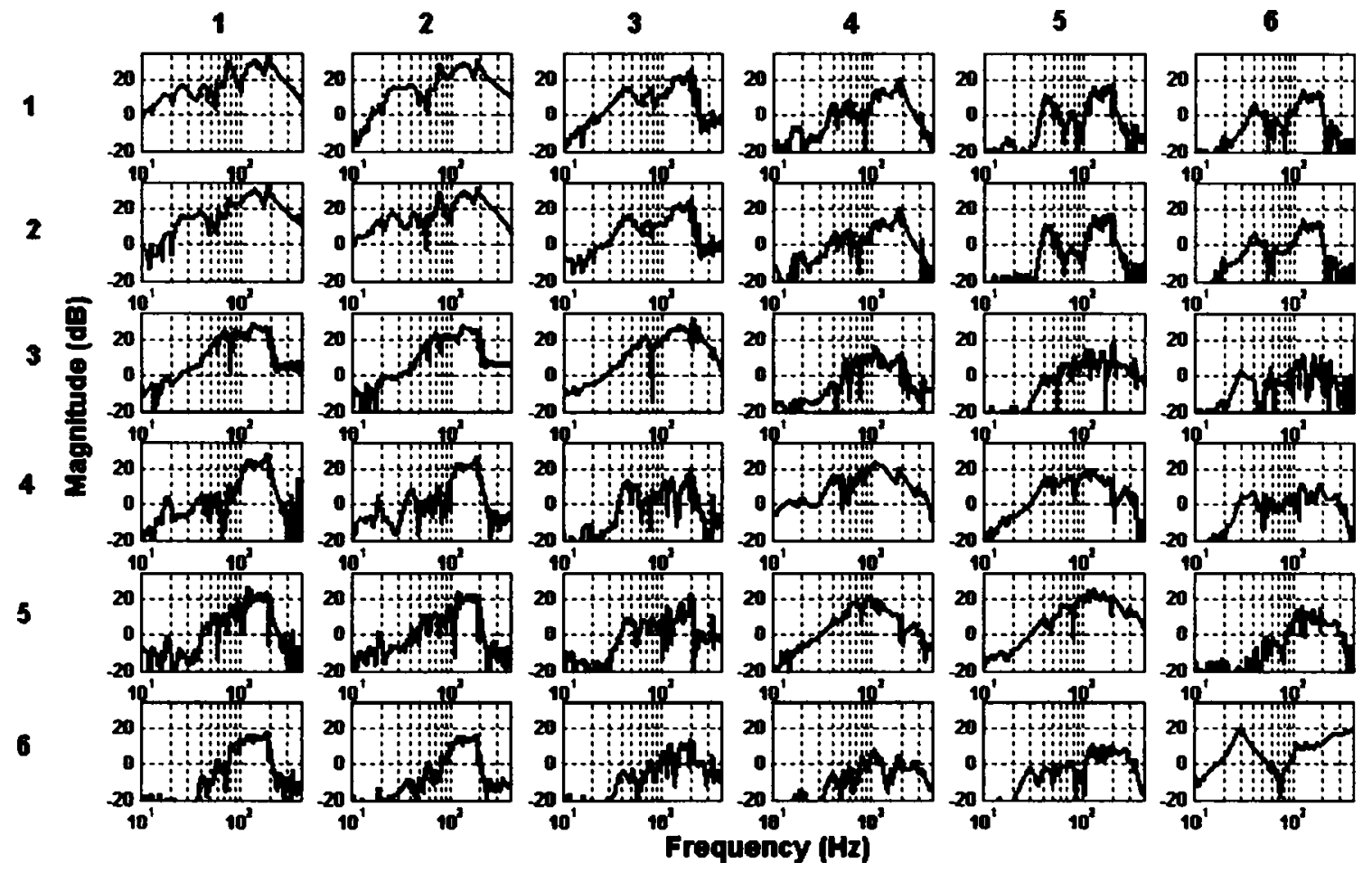

FIG. 5. Frequency response (accelerometer over piezo drive) of the original system. Rows are piezo drive axes; columns are accelerometer axes.

erometer signal. Therefore, the slope of magnitude response at the low frequency range should represent the $40 \mathrm{~dB} /$ decade characteristics.

\section{CONTROLLER DESIGN}

After diagonalizing the system, we need only consider the diagonal terms. Consider the time-invariant, linear, continuous-time, SISO system with uncertainties and exogenous disturbances,

$$
\begin{aligned}
& \dot{x}(t)=(A+\Delta A) x(t)+(B+\Delta B) u(t)+f(t), \\
& y(t)=(C+\Delta C) x(t)+v(t),
\end{aligned}
$$

where $x(t) \in \mathfrak{R}^{n}$ and $u(t) \in \mathfrak{R}^{1}$ are the state and the control input variables respectively; $y(t) \in \mathfrak{R}^{1}$ is the measured system output, $f(t) \in \mathfrak{R}^{n}$ is the exogenous disturbance, and $v(t) \in \mathfrak{R}^{1}$ is the sensor noise $A, B$, and $C$, denote the nominal or known system matrices, and $\Delta A, \Delta B, \Delta C$ are the respective matrix uncertainty components. Choose the sliding sur-

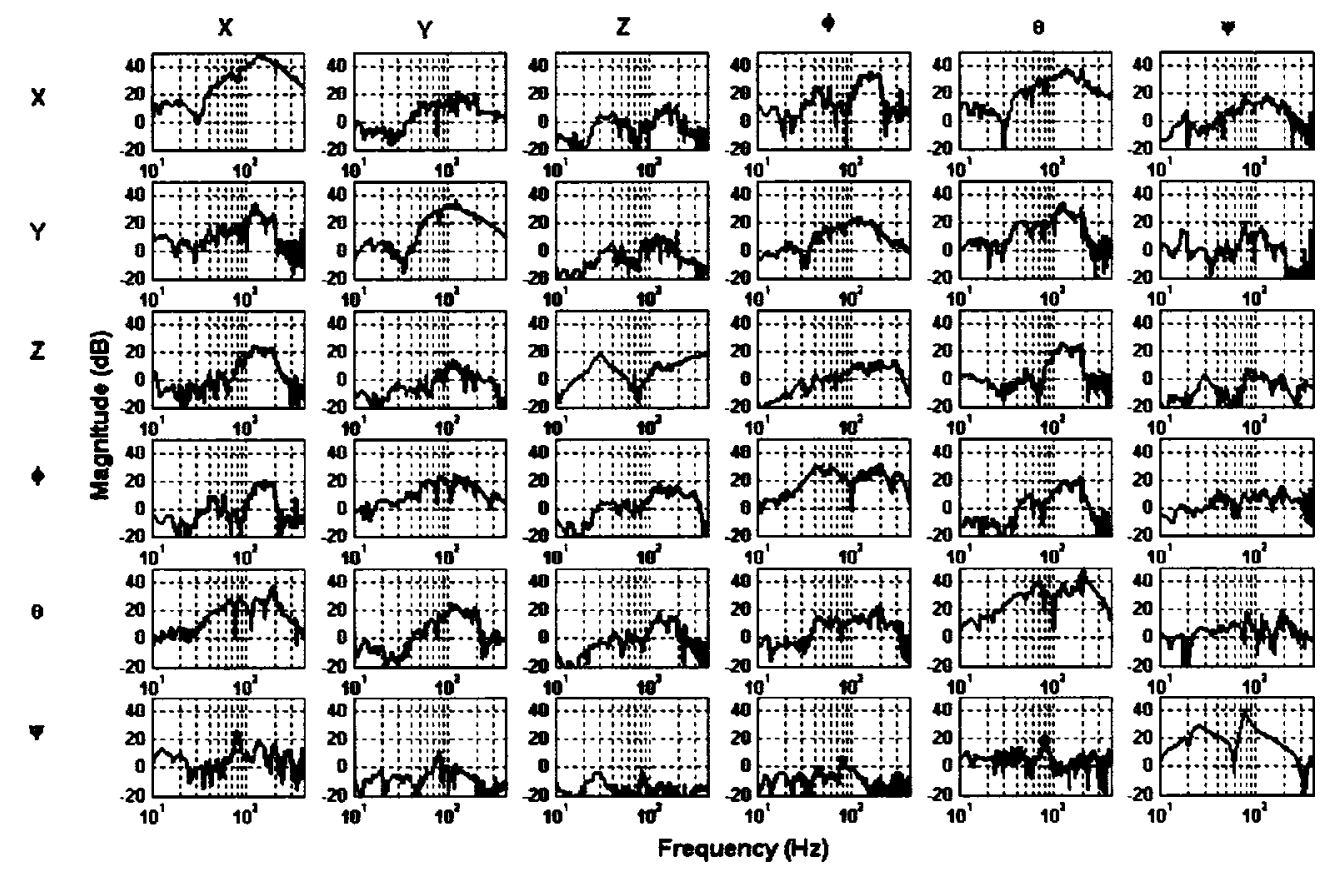

FIG. 6. Frequency response (accelerometer over piezo drive) of the diagonalized system. Rows are piezo drive axes; columns are accelerometer axes. 

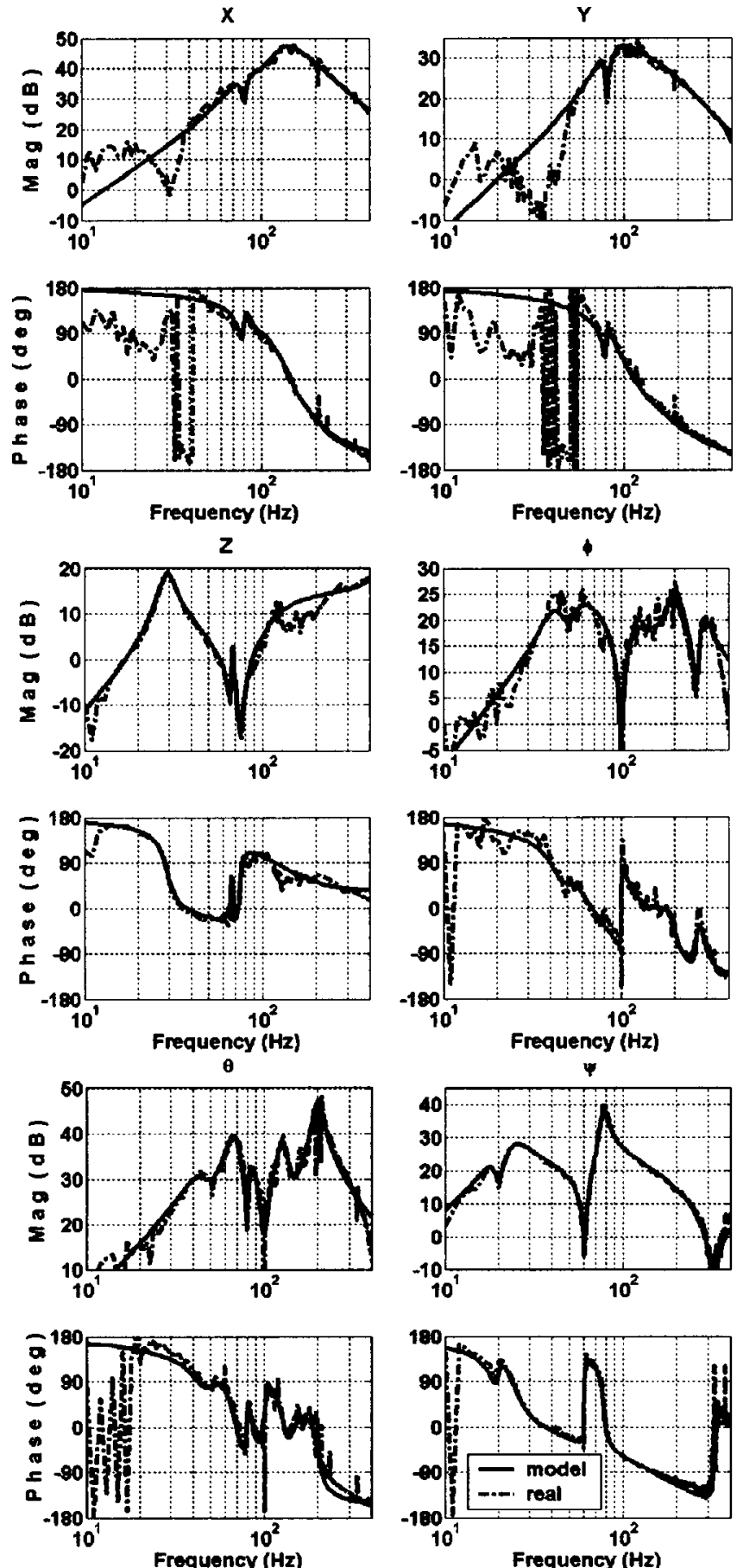

FIG. 7. System identification comparison between curve fit model and real system for the diagonalized sytem. $X$ is the frequency response of the diagonal entries from actuator $X$ to accelerometer $\mathrm{X} ; \mathrm{Y}$ is frequency response of the diagonal entries from actuator $\mathrm{Y}$ to accelerometer $Y$.

face $s(t) \in \mathfrak{R}^{1}$ and the control effort as in Eqs. (17) and (18),

$$
\begin{aligned}
& s(t)=\Lambda x(t), \\
& u(t)=-(\Lambda B)^{-1} \Lambda A_{x}(t)-[\alpha|x(t)|+\delta] \operatorname{sign}(s(t)) \quad a, \delta>0,
\end{aligned}
$$

where $|x(t)|=\sum_{i-1}^{n}\left|x_{1}\right| \in \mathfrak{R}^{1}$ is the sum of absolute value of each state. The row vector $\Lambda \in \mathfrak{R}^{1}{ }^{x n}$ determines the dynamic trajectory of the states on the sliding manifold. $\alpha$ and $\delta$ are adjustable constants. In order to check whether or not the

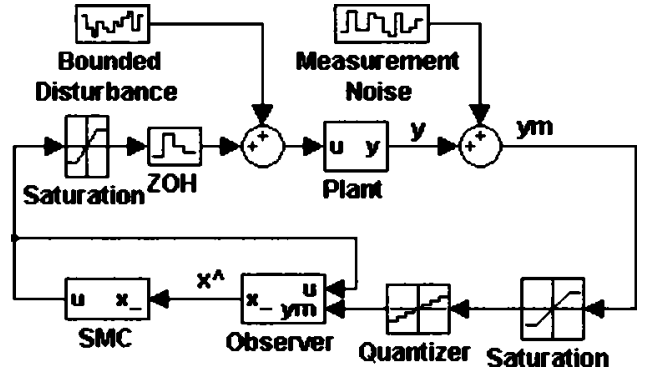

FIG. 8. Computer simulation for SMC.

states will converge to the sliding manifold, we need to check for stability. We choose the Lyapunov function to be

$$
V(t)=0.5 s(t)^{T} s(t) .
$$

One can find the time derivative of the Lyapunov function to be

$$
\begin{aligned}
\dot{V}(t)= & s(t)^{T}\left[\Lambda \Delta A x(t)-\Lambda \Delta B(\Lambda B)^{-1} \Lambda A x(t)-\Lambda(B\right. \\
& +\Delta B) \alpha|x(t)| \operatorname{sign}(s(t))-\Lambda(B+\Delta B) \delta \operatorname{sign}(s(t)) \\
& +\Lambda f(t)], \\
\dot{V}(t)= & s(t)^{T} \Lambda\left|\Delta A-\Delta B(\Lambda B)^{-1} \Lambda A\right| x(t)-\Lambda(B \\
& +\Delta B) \alpha|x(t)||s(t)|-\Lambda(B+\Delta B) \delta|s(t)|+s(t)^{T} \Lambda f(t) .
\end{aligned}
$$

If the conditions $\quad \Lambda\left\lfloor\Delta A-\Delta B(\Delta B)^{-1} \Lambda A\right\rfloor x(t)<\Lambda(B$ $+\Delta B) \alpha|x(t)|$ and $\Lambda(B+\Delta B) \delta>\Lambda f(t)$ are true, the condition $\dot{V}(t)<0$ is true. The state will converge to a sliding surface. The constant $\alpha$ can accommodate parameter uncertainties $(\Delta A, \Delta B)$ and $\delta$ can handle the disturbance $f(t)$. Using this controller, a small $\alpha$ is sufficient to overcome small parameter uncertainties. Once the state is on the sliding manifold, the dynamics depend on the vector $\Lambda$. One can use the optimal control technique to choose a suitable value of vector $\Lambda$. Assuming $\Delta B$ is zero, one can convert the nominal system into a system with a state $z(t)=\left[\begin{array}{ll}z_{1}(t) & z_{2}(t)\end{array}\right]^{T}$,

$$
\begin{aligned}
& \dot{z}_{1}(t)=A_{11} z_{1}(t)+A_{12} z_{2}(t), \\
& \dot{z}_{2}(t)=A_{21} z_{1}(t)+A_{22} z_{2}(t)+u(t),
\end{aligned}
$$

where $z_{1}(t) \in \mathfrak{R}^{n-1}$ and $z_{2}(t) \in \mathfrak{R}^{1}$ are the system states, and $A_{11} \in \mathfrak{R}^{(n-1) x(n-1)}, A_{12} \in \mathfrak{R}(n-1) x 1, A_{21} \in \mathfrak{R}^{1 x(n-1)}$, and $A_{22}$ $\in \mathfrak{R}^{1 \times 1}$ are system matrices. It is now possible to choose a sliding surface of the form $S(t)=K z_{1}(t)+z_{2}(t)$, and

$$
\Lambda=\left[\begin{array}{ll}
K & 1
\end{array}\right] \text {. }
$$

After the state converges to the sliding manifold, the state will stay on the manifold, i.e.,

$$
s(t)=0 \Rightarrow z_{2}(t)=-K z_{1}(t) .
$$

Choose the following criterion: ${ }^{9}$

$$
J(t)=\int_{0}^{\infty}\left[z_{1}(t)^{T} Q z_{1}(t)+z_{2}(t)^{T} R z_{2}(t)\right] d t
$$

to be minimized, where $R$ and $Q$ are positive definite and positive semidefinite matrices to be determined by choosing 


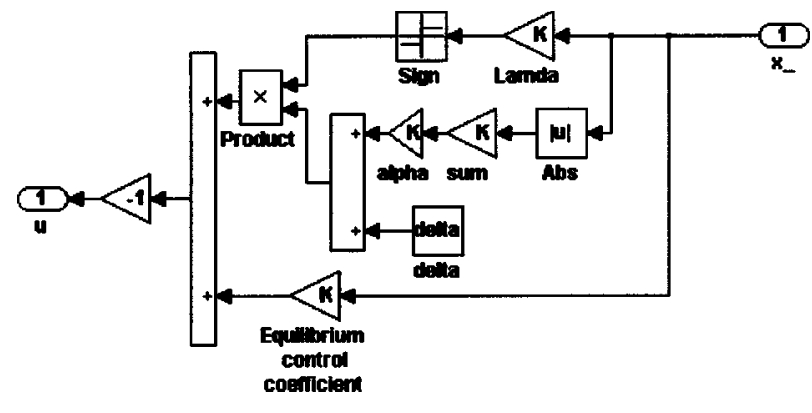

FIG. 9. The structure of SMC block.

on the relative importance of the states $z_{1}(t)$ and the states $z_{2}(t)$. The row vector $K$ can be selected by solving the Algebraic Riccati Equation (ARE).

\section{SIMULATION RESULTS}

The simulation is based on the complete system model. The controller design, however, is based on the linearized model. Figure 8 is the simulation setup for the SMC control system. This simulation was done in Simulink. ${ }^{10}$ (Commercial equipment and materials are identified in order to adequately specify certain procedures. In no case does such identification imply recommendation or endorsement by the National Institute of Standards and Technology, nor does it imply that the materials or equipment identified are necessarily the best available for the purpose.) The controller also includes a state estimator. The simulation includes the effects of the digital signal processor (DSP) implementation. The quantizer and saturation limits simulate the ADC; the saturation and Zero-Order-Hold $(\mathrm{ZOH})$ simulate the DAC device. Figure 9 is the structure of the SMC with piecewise linear discontinuous control, which is implemented by Eq. (18). The equilibrium control coefficient matrix is $-(\Lambda B)^{-1} \Lambda A$. To optimize the performance, one starts by tuning the observer parameter for the best estimation, and then one tunes the SMC parameters with the real system states in simulation. The part are then combined to examine the overall system performance.

Figure 10 is the power spectrum comparison with and without the SMC controller for the $x$-axis Accelerometer.

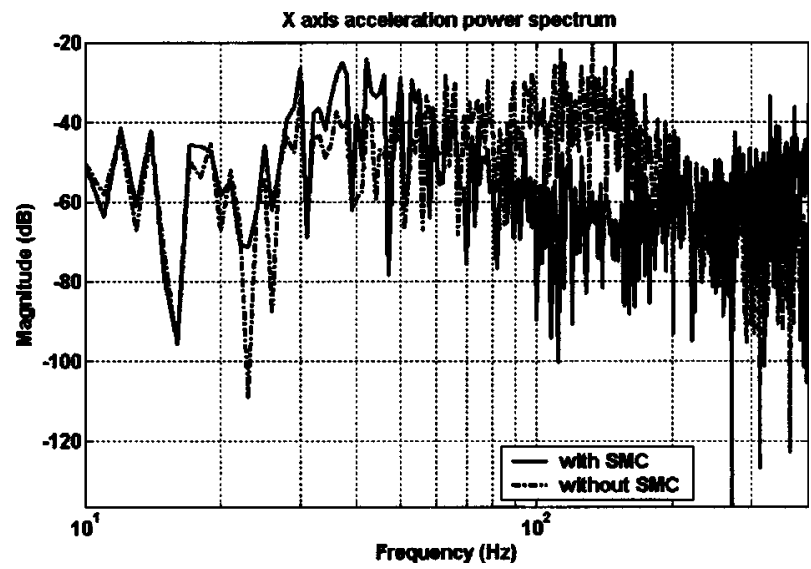

FIG. 10. Power spectrum of $\mathrm{x}$-axis acceleration with an without SMC controller.

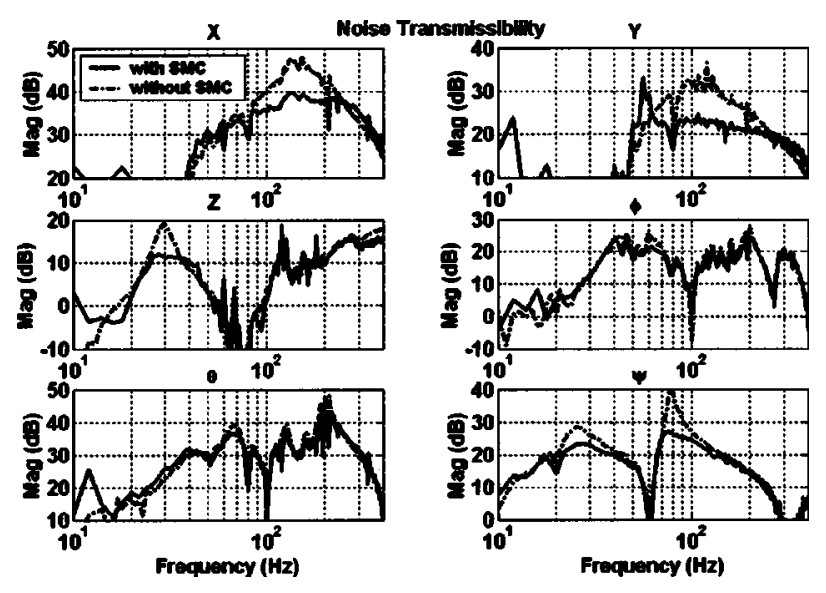

FIG. 11. Noise transmissibility with and without SMC.

There is a good attenuation in the $100 \mathrm{~Hz}$ to $200 \mathrm{~Hz}$ range. There is also a little bit more high-frequency content caused by chattering.

\section{EXPERIMENTAL RESULT}

The SMC controller has been applied to the physical Mallock suspension system. The noise transmissibility with and without the SMC is shown in Fig. 11. The control system achieves better noise attenuation response in the low frequency region for actuators $X$ and $Y(100 \mathrm{~Hz}$ to $200 \mathrm{~Hz})$. One observes very small magnitudes of actuators $X$ and $Y$ at the low frequency. Therefore, the signal to noise ratio is also very small. The measurement data in that region is not eliable. One also observes more high frequency noise (above $250 \mathrm{~Hz}$ ) due to the chattering phenomena. Figure 12 shows the actual attenuation effects when sinusoidal disturbances are injected into each channel. The controller is turned on at a delayed time to inspect the control effect. One clearly observes the attenuation effects in channels $x, y, z$, and $\psi$. The $\phi$ and $\theta$ directions are not shown because the improvements are not significant.

Figure 13 shows acquired SPM images with an injected fixed sine disturbance. It is clear that the disturbance signal has introduced as alias fluctuation in the profile. With the
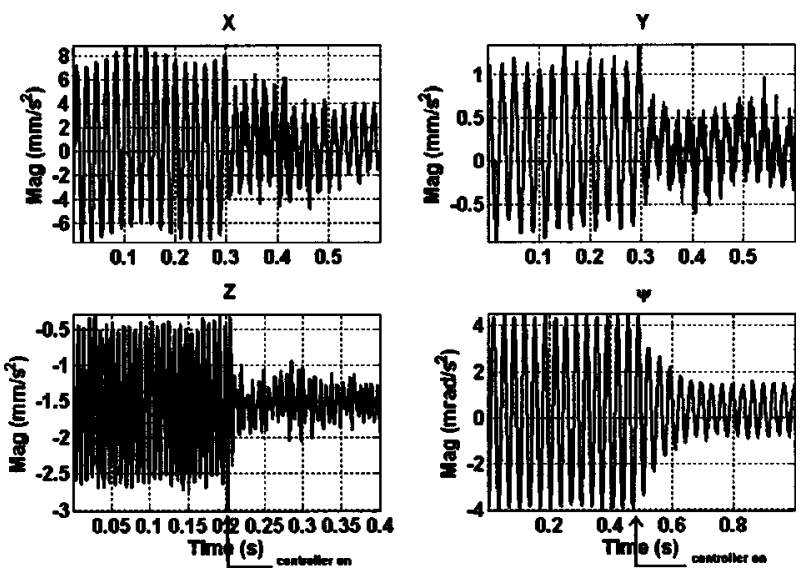

FIG. 12. Acceleration signals are shown for fixed-frequency sine wave disturbances. The controller is off in the beginning and then is turned on after a certain time. 

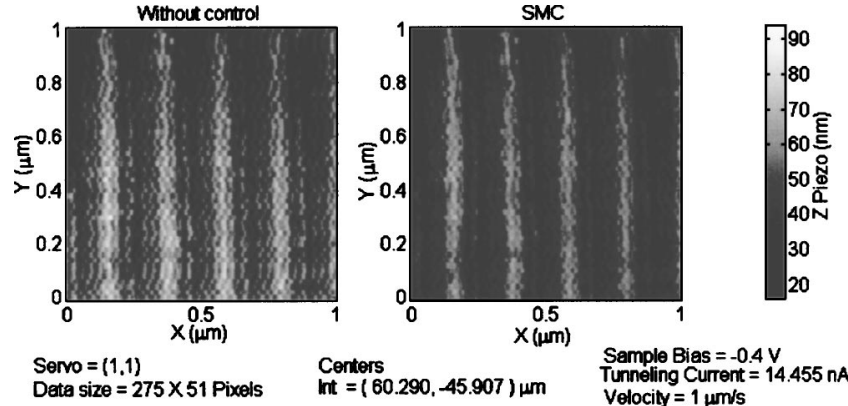

Servo $=(1.1)$
Data size $=275 \times 51$ Pixels

Centers Centers $=(60.290,45.907) \mathrm{um}$ Velocily $=1 \mathrm{um} / \mathrm{s}$

FIG. 13. $\mathrm{M}^{3}$ image with a fixed sine disturbance (no active vibration control and SMC vibration control).

SMC in operation, the image is more immune to the disturbance. The system maintains high resolution with the controller even when the scanning is set at a relatively high scanning speed $(1 \mu \mathrm{m} / \mathrm{s})$.

\section{FUTURE WORKS}

It is clear by now that the environment vibration imposes limitation on the achievable resolution of the SPM measurement. The accelerometer resolution determines the ability of the active vibration isolation system. Future research will put emphasis on enhancing the signal-to-noise ratio of the sensor measurement. The goal is for the $\mathrm{M}^{3}$ system to achieve subangstrom measurement.

\section{ACKNOWLEDGMENTS}

Technical assistance from Bill Penzes, Geroge Witzgall, and Vince Scheuerman are gratefully acknowledged.

${ }^{1}$ J. Kramar, E. Amatucci, D. Gilsinn, J. Jun, W. Penzes, F. Scire, E. C. Teague, and J. Villarrubia, SPIE Conference on Metrology, Inspection, and Process Control for Microlithography XIII, Santa Clara, California, 1999, pp. 1017-1028.

${ }^{2}$ E. C. Teague, J. Vac. Sci. Technol. B 7, 1898 (1989).

${ }^{3}$ D. Gilsinn, M. Lyons, F. Scire, E. C. Teague, E. Amatucci, and T. McIntyre, Draft National Institute of Standards and Technology Interagency Report 1991 (unpublished).

${ }^{4}$ E. Anderson, D. Leo, and M. Holcomb, in Proceedings of the 19th Annual AAS Guidance and Control Conference, Breckenridge, CO, 1996.

${ }^{5}$ Z. Geng and L. Hayes, IEEE Trans. Control Syst. Technol. 2, 45 (1994).

${ }^{6}$ D. Thayer, J. Vagners, A von Flotow, C. Hardham, and K. Scribner, in Proceedings of the 21st Anneal AAS Guidance and Control Conference, Breckenridge, Colorado, (1998), pp. 497-506.

${ }^{7}$ R. S. Whipple, Trans. Opt. Soc., London 22, 48 (1920).

${ }^{8}$ K. J. Lan, J. Y. Yen, and J. A. Kramear, Asian J. Control 6, 100 (2004).

${ }^{9}$ F. L. Lewis and V. L. Syrmos, Optimal Control, 2nd ed (Wiley, New York, 1976), p. 161-196

${ }^{10}$ J. Little and C. Moler, computer code MATLAB (MathWorks, Inc., Natick, MA, 1984). 